\title{
Irreducible nonlocality of optical model potentials based on realistic $N N$ interactions
}

\author{
H. F. Arelland* \\ Department of Physics - FCFM, University of Chile, \\ Av. Blanco Encalada 2008, Santiago, Chile and \\ CEA,DAM,DIF, F-91297 Arpajon, France \\ G. Blanchon \\ CEA,DAM,DIF, F-91297 Arpajon, France
}

(Dated: March 15, 2022)

\begin{abstract}
We investigate the nonlocal structure of optical model potentials for nucleon-nucleus scattering based on microscopic approaches. To this purpose, in-medium folding optical potentials are calculated in momentum space and their corresponding coordinate-space counterpart are examined, paying special attention to their nonlocal shape. The nucleon-nucleon effective interaction consists of the actual full off-shell $g$ matrix in Brueckner-Hartree-Fock approximation. The nonlocality of effective interactions is preserved throughout all stages in the the calculation. Argonne $v_{18}$ bare potential and chiral next-to-next-to-next-to-leading order bare interaction are used as starting point. The study is focused on proton elastic scattering off ${ }^{40} \mathrm{Ca}$ at beam energies between 30 and $800 \mathrm{MeV}$. We find that the gradual suppression of high-momentum contributions of the optical potential results in quite different-looking coordinate-space counterparts. Despite this non-uniqueness in their nonlocal structure, the implied scattering observables remain unchanged for momentum cutoff above a critical one, which depends on incident energy of the projectile. We find that coordinate-space potentials with momentum cutoffs at the critical value yield the least structured nonlocal behavior. Implications of these findings are discussed.
\end{abstract}

PACS numbers: 24.10.Ht 25.60.Bx 25.40.Cm

\section{INTRODUCTION}

The inclusion of nonlocalities in phenomenological optical model potentials for nucleon-nucleus $(N A)$ scattering, pioneered in the early 60 s by Perey and Buck [1, made possible simple and robust phenomenological accounts of elastic scattering data for targets with nuclear masses in the range $27 \leq A \leq 208$, and nucleon energies of up to $30 \mathrm{MeV}$. From a microscopic point of view, considering that the in-medium density-dependent nucleonnucleon $(N N)$ effective interaction is intrinsically nonlocal, it is natural to expect that the optical potential itself would turn a nonlocal operator. Moreover, even under the simplifying assumption that effective interaction between nucleons was local, nonlocal contributions to the optical potential would arise from the exchange term after the antisymmetrization of the interaction. As we shall demonstrate, for a given optical potential in momentum space there is a whole class of potentials in coordinate space with different nonlocal structure which yield the same scattering observables. The question that comes out then is up to what extent the nonlocal features exhibited by microscopic $N A$ optical model potentials are unique so as to be inferred from ab-initio approaches.

Microscopic models for the optical potential provide us with a direct link between the elementary $N N$ bare interaction and the many-body physics involved in $N A$ scattering phenomena [2 5]. The fermionic character of

\footnotetext{
*arellano@dfi.uchile.cl
}

all $A+1$ constituents together with the nonlocal structure of the internucleon effective interaction result into a nonlocal coupling between single nucleons with the remaining $A$ nucleons. Several efforts have been made in the past decades aimed to construct these potentials using realistic $N N$ bare interactions to model effective interactions. Such is the case of folding models, where a convolution takes place between an effective interaction (off-shell $g$ or free $t$ matrix, depending on the energy of application) and the ground-state mixed density of the target 4,6 , 8 . Along this line, momentum- and coordinate-space approaches adopted by different groups have evolved on parallel tracks, resulting on comparable description of available scattering data. In practice, however, specific considerations in the construction and treatment of the effective interaction make different momentum- from coordinate-space approaches. The main difference among them lies in the fact that in the former the actual fully off-shell $g$ (or $t$ ) matrix is folded with the target mixed density, whereas in the latter the $g$ matrix is first localized and then folded. By suppressing nonlocalities in the $N N$ effective interaction (such as Melbourne 6] or Hamburg 9]) nonlocalities in the optical potential arise exclusively from the exchange contribution. This feature contrasts with folding models in momentum space [8, 10,13], where both direct and exchange terms result nonlocal.

Another well established microscopic approach for the $N A$ optical model, particularly at low energies, is the one developed by Mahaux and collaborators [3], coined as local density approximation for the optical potential. Here the optical potential for nucleon scattering from a finite 
nucleus becomes local by construction, where at each coordinate $r$ of the projectile in the nucleus it is mapped the on-shell mass operator from infinite nuclear matter, evaluated at a density equal to that of the target at coordinate $r$, namely $\rho(r)$. The energy at which the mass operator is evaluated corresponds to that of the beam. Besides the fact that the resulting optical potential is local, its spin-orbit part becomes undefined. This last limitation is fixed with the introduction of a phenomenological spin-orbit coupling. Studies are now being pursued to incorporate a nonlocal absorptive contributions to the dispersive optical model within the dispersive self-energy method [14].

In this work we study the nonlocal structure of microscopic optical model potentials considering proton scattering off ${ }^{40} \mathrm{Ca}$ at beam energies between 30 and $800 \mathrm{MeV}$. The potentials are calculated in momentum space making use of the density-dependent BruecknerHartree-Fock $g$ matrix [7, 13] where its exact nonlocal and off-shell structure are retained. Once these potentials are transformed into coordinate space considering different cutoffs at high momenta, we assess their nonlocal structure as well as equivalence in the context of scattering observables. We show that the issue of the non-uniqueness of the nonlocality is possibly solved considering that different looking potentials turn out to share a similar shape when a non-reducible momentum cutoff is adopted.

This article is organized as follows. In Sec. II we describe our framework and analyze the case of $p+{ }^{40} \mathrm{Ca}$ elastic scattering at $200 \mathrm{MeV}$. We evaluate the potential in momentum space and investigate its structure in coordinate space when the high momentum components are suppresses, with focus on its nonlocal structure. In Sec. III we discuss the existence of a minimum cutoff applied to optical potentials in momentum space, above which all scattering observables remain the same. We explore the nonlocality with those of Perey-Buck-type [15] as well as that from a microscopic optical potential obtained with the Nuclear Structure Method [16] based on Green's function formalism in the Random-Phase Approximation using Gogny's effective interaction. In Sec. IV we summarize and discuss the main results of this work.

\section{PROTON-NUCLEUS SCATTERING: A STUDY CASE}

Consider the scattering of protons off ${ }^{40} \mathrm{Ca}$ at beam energy $E$. The optical model potential in momentum space can be expressed as the sum of its central and spinorbit contributions in the form

$$
\tilde{U}\left(\boldsymbol{k}^{\prime}, \boldsymbol{k} ; E\right)=\tilde{U}_{c}\left(\boldsymbol{k}^{\prime}, \boldsymbol{k} ; E\right)+i \boldsymbol{\sigma} \cdot \hat{n} \tilde{U}_{s o}\left(\boldsymbol{k}^{\prime}, \boldsymbol{k} ; E\right),
$$

with $\boldsymbol{\sigma}$ denoting the spin of the projectile and $\hat{n}$ the unit vector perpendicular to the scattering plane defined by $\hat{n}=\left(\boldsymbol{k}^{\prime} \times \boldsymbol{k}\right) /\left\|\boldsymbol{k}^{\prime} \times \boldsymbol{k}\right\|$. This same operator is often denoted as $\tilde{\Sigma}\left(\boldsymbol{k}^{\prime}, \boldsymbol{k} ; E\right)$ or $\tilde{M}\left(\boldsymbol{k}^{\prime}, \boldsymbol{k} ; E\right)$ in the literature [2, 17]. The optical potential is evaluated in momentum representation following procedures outlined in Ref. 7], where an in-medium effective interaction is folded with the target full mixed density. The (nonlocal) densitydependent $N N$ effective interaction is taken as the actual off-shell $g$ matrix solution of the Brueckner-BetheGoldstone equation in the Brueckner-Hartree-Fock approximation. In the absence of medium effects the $g$ matrix turns the free $t$ matrix, resulting in the impulse approximation for the optical model potential in multiplescattering expansion [10]. An appealing attribute of the momentum-space folding approach we follow is that it enables, within a single framework, parameter-free descriptions of nucleon scattering off nuclei at energies ranging from few tens of $\mathrm{MeV}$ up to $1 \mathrm{GeV}$ [7, 13, 18, feature not shared by any other reported approach.

To obtain the $g$ matrix we make use of the traditional Argonne $v_{18}$ [19] (AV18) bare potential which has been fit to $N N$ phase-shift data at beam energies below pion production threshold, together with static properties of the deuteron. We also include in this study a chiral effectivefield-theory (EFT) interaction, based on chiral perturbation theory. The resulting bare interaction is constructed with nucleons and pions as degrees of freedom, with the two-nucleon part $(2 N)$ fit to $N N$ data. We consider the chiral $2 N$ force up to next-to-next-to-next-to-leading order (N3LO) given by Entem and Machleidt [20. To each of these interactions we have calculated their corresponding infinite nuclear matter single-particle selfconsistent fields following Refs. [21 23, to subsequently be used to obtain fully off-shell $g$ matrices. Radial proton and neutron densities for the ${ }^{40} \mathrm{Ca}$ target are obtained from Hartree-Fock-Bogoliubov calculations based on the finite range, density-dependent Gogny's D1S interaction 24.

\section{A. Momentum- vs coordinate-space structure}

Typically, momentum-space NA potentials are evaluated at relative momenta of up to about 10 to $15 \mathrm{fm}^{-1}$ -depending on the beam energy- choosing an angular mesh $\hat{k} \cdot \hat{k}^{\prime}$ suitable for reliable partial wave expansions. Once the central and spin-orbit components of $\tilde{U}\left(\boldsymbol{k}^{\prime}, \boldsymbol{k} ; E\right)$ are obtained we extract their corresponding partial wave components $\tilde{U}_{l}\left(k^{\prime}, k\right)$, with $l$ the orbital angular momentum.

Let us first consider $p+{ }^{40} \mathrm{Ca}$ elastic scattering with proton beam energy of $200 \mathrm{MeV}$. In Figs. 1 and 2 we show surface plots of the real (a) and imaginary (b) $s$-wave components of the (central) optical potential, $k^{\prime} \tilde{U}_{c}\left(k^{\prime}, k\right) k$, as functions of the relative momenta $k$ and $k^{\prime}$. Fig. 1 and 2 are based on AV18 and N3LO bare potentials, respectively. The short segments on each sheet denote the on-shell momentum. As observed, both real and imaginary components exhibit their dominant contributions along a diagonal band. In the case of the real 

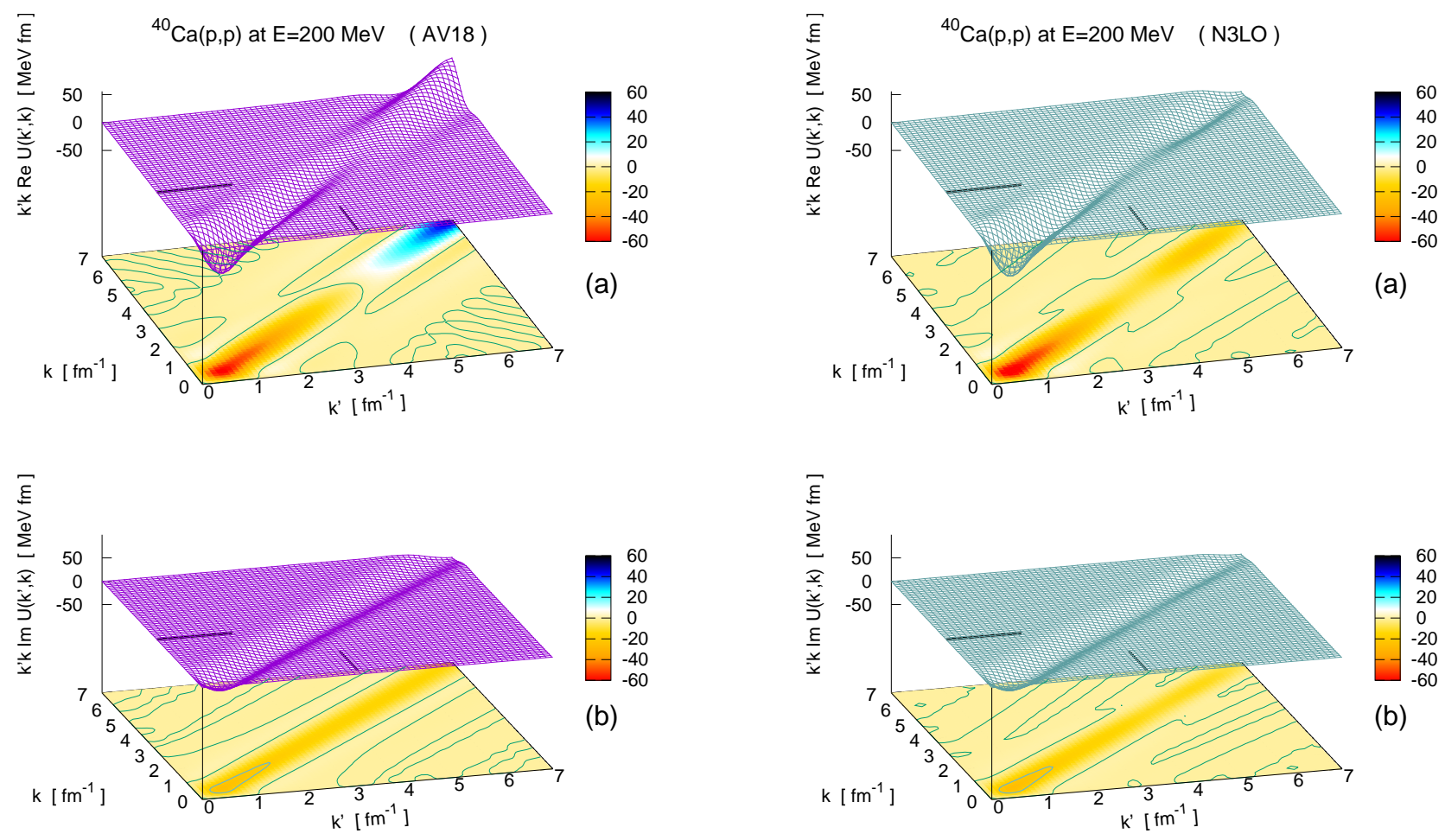

FIG. 1. Real (a) and imaginary (b) parts of the $s$-wave central optical potential as functions of the relative momenta $k$ and $k^{\prime}$. Potential corresponding to $p+{ }^{40} \mathrm{Ca}$ scattering at $200 \mathrm{MeV}$. Results based on AV18 bare interaction. Color code in units of $\mathrm{MeV}$ fm.

component based on AV18, a change of sign takes place in the vicinity of $k=k^{\prime} \approx 4 \mathrm{fm}^{-1}$. This feature contrasts with that in panel $a$ of Fig. 2, where the real part of the potential based on N3LO is weaker at high momenta, without change of sign. In the case of the imaginary component, both bare interactions yield an $s$-wave potential mostly negative maintaining its sign along the diagonal.

In coordinate space the central part of the potential reads

$U_{l}\left(r^{\prime}, r\right)=\frac{2}{\pi} \int_{0}^{\infty} k^{\prime 2} d k^{\prime} \int_{0}^{\infty} k^{2} d k j_{l}\left(k^{\prime} r^{\prime}\right) \tilde{U}_{l}\left(k^{\prime}, k\right) j_{l}(k r)$,

resulting a nonlocal function in the general case (we omit subscript $c$ for simplicity). A similar expression holds for the spin-orbit term. Actual evaluations of the above integrals are performed up to some upper momenta chosen to guarantee convergence of the evaluated scattering observables. To select the momentum up to which integrals over $k$ are done we introduce a cutoff function $f_{\Lambda}(k)$ such that

$$
\tilde{U}\left(k^{\prime}, k\right) \rightarrow \tilde{U}_{\Lambda}\left(k^{\prime}, k\right)=f_{\Lambda}\left(k^{\prime}\right) \tilde{U}\left(k^{\prime}, k\right) f_{\Lambda}(k),
$$

FIG. 2. The same as Fig. 1] but for N3LO interaction.

where

$$
f_{\Lambda}(k)=\frac{1}{2}\left[1-\tanh \left(\frac{k-\Lambda}{\delta}\right)\right] .
$$

For sufficiently small $\delta$ this function resembles the Heavyside step function $\Theta(\Lambda-k)$. Hence, $\Lambda$ defines the momentum above which momenta in $\tilde{U}\left(k^{\prime}, k\right)$ are suppressed. Under this definition $\delta$ represents the width of the cutoff, which for this work we have chosen equal to $0.2 \mathrm{fm}^{-1}$ throughout. This cutoff function plays an analogous role to momentum-space regulators in renormalization group transformations [25].

For a given beam energy $E$ we have performed scattering calculations in coordinate space considering various values of $\Lambda$, obtaining practically the same observables when $\Lambda$ lies above certain critical cutoff momentum. At the same time, however, different choices of $\Lambda$ above this critical momentum lead to potentials with quite different nonlocal structure, feature to be addressed in more detail next.

On each of Figs. 3 and 4 we show six contour plots in the $\left(r, r^{\prime}\right)$ plane for the calculated $r^{\prime} U\left(r^{\prime}, r\right) r$, with three choices of $\Lambda$ at and above $4 \mathrm{fm}^{-1}$. These choices lead to the same scattering observables, as will be discussed later. Figs. 3 and 4 are based on AV18 and N3LO bare interactions, respectively. Panels $a, b$ and $c$ on the lefthand side (l.h.s.) correspond to the real component of the 

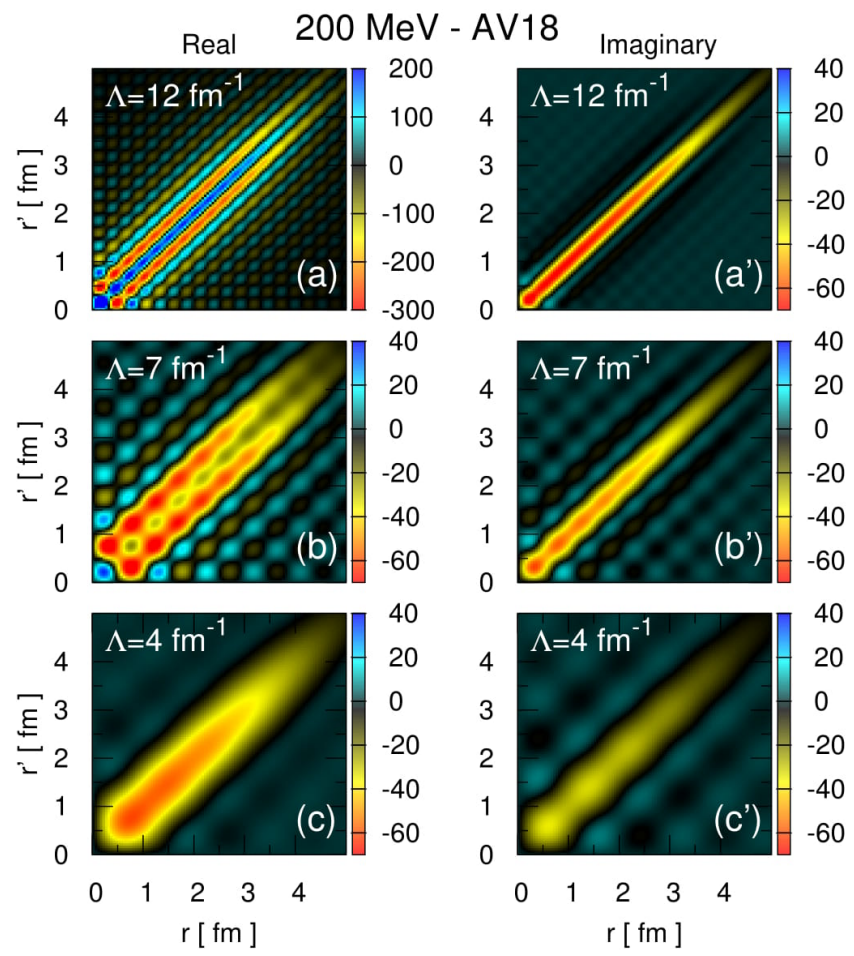

FIG. 3. Contour plots for the real (l.h.s. panels) and imaginary (r.h.s. panels) $s$-wave central optical potential as functions of the relative distance $r$ and $r^{\prime}$. Potential for $p+{ }^{40} \mathrm{Ca}$ scattering at $200 \mathrm{MeV}$. Results based on AV18 bare interaction. Frames a, b and c represent $N A$ potentials with cutoffs at $\Lambda=12,7$ and $4 \mathrm{fm}^{-1}$, respectively. All three potentials yield the same scattering observables. Color bar in units of $\mathrm{MeV} \mathrm{fm}^{-1}$.

potential, while the prime-labeled panels on the righthand side (r.h.s.) represent the imaginary part. Panels $a$ and $a^{\prime}$ show results for $\Lambda=12 \mathrm{fm}^{-1}, b$ and $b^{\prime}$ for $\Lambda=7 \mathrm{fm}^{-1}$, and $c$ and $c$ ' for $\Lambda=4 \mathrm{fm}^{-1}$. What is evidenced in panel $a$ of Fig. 3 is how strong and rugged appears the real part of the AV18-based potential in coordinate space, as inferred from the narrow diagonal bands of opposite signs (note the $-300: 200$ scale for this panel). Observe also the narrow oscillatory pattern taking place along transverse lines, i.e. those where $\left(r+r^{\prime}\right)$ is constant. The corresponding imaginary component shown in panel $a^{\prime}$ is less intense, with its sharp dominant contributions located near the diagonal $r=r^{\prime}$. It is worth stating that if the potential was local, its plot on any of the panels should result in a narrow band along the diagonal $r=r^{\prime}$. As the cutoff $\Lambda$ diminishes the potential in coordinate space becomes less structured, though it retains its nonlocal nature. This general feature is evidenced in panels $c$ and $c^{\prime}$ of Figs. 3 and 4 for $\Lambda=4 \mathrm{fm}^{-1}$.

Clearly the momentum cutoff $\Lambda$ has an incidence on the shape of the potential in coordinate space, particularly with regard to its nonlocality. For a closer scrutiny of this feature, in Figs. 5 (for AV18) and 6 (for N3LO) we plot $r^{\prime} U\left(r^{\prime}, r\right) r$ as functions of the difference $\left(r^{\prime}-r\right)$ along transverse lines where $r+r^{\prime}$ is kept constant. Black, blue
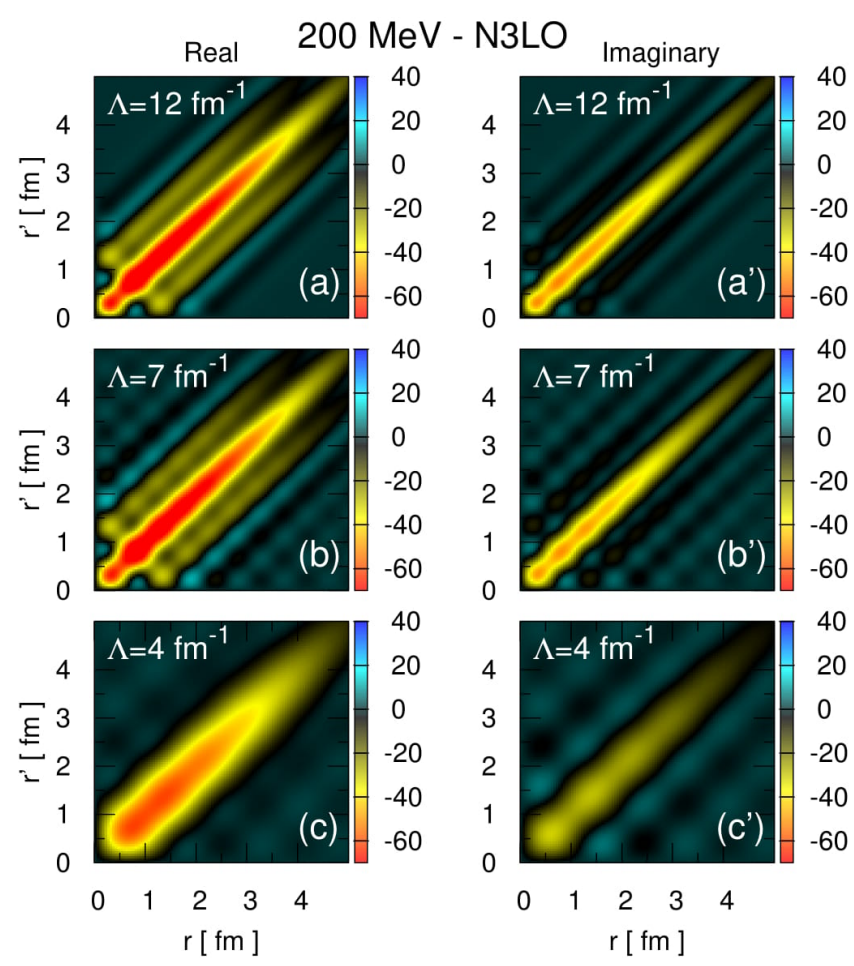

FIG. 4. The same as Fig. 3, but for N3LO bare interaction.

and red curves correspond to $\Lambda$ equal to 12,7 and $4 \mathrm{fm}^{-1}$, respectively. Panels $a$ and $b$ on the l.h.s. represent $r^{\prime} U\left(r^{\prime}, r\right) r$ in the case $\left(r+r^{\prime}\right) / 2 \equiv R_{A}$, with $R_{A}=2 \mathrm{fm}$. When $r \approx r^{\prime}$, this choice of $R_{A}$ represents a region near the bulk of the nucleus. Panels $c$ and $d$ on the r.h.s. correspond to case $R_{A}=4 \mathrm{fm}$, near the surface of the nucleus. Upper (lower) panels show the real (imaginary) part of the potential. We observe that the real part oscillates as a function of $r^{\prime}-r$, being stronger for $r=r^{\prime}$, and decreasing as $\left|r^{\prime}-r\right|$ increases. The imaginary part behaves similar to the real part, but featuring more damped oscillations. When comparing l.h.s with r.h.s. panels, we observe that the potential weakens at the surface.

Any near local potential would resemble some representation of the Dirac $\delta$ function of finite width along transversal lines. The curvature at $r^{\prime}=r$, would serve to gauge the nonlocality at $R_{A}=r$. From the plots shown in Figs. 5 and 6 we observe a clear sensitivity of the curvature of the potential at $r=r^{\prime}$, under the three values of $\Lambda$, evidencing different nonlocalities among them. What emerges as an interesting property is that as $\Lambda$ diminishes, the coordinate-space optical potential based on AV18 and N3LO become similar, feature we illustrate more closely in Fig. 7 for the $s$-wave component. Here blue and red curves represent results based on AV18 and N3LO bare interactions, respectively. Solid (dashed) curves correspond to the real (imaginary) component of the potential. Labels for $R_{A}$ identify regions in the nucleus at which the potential is plotted. Observe that the real component of the potential is slightly more intense for N3LO than for AV18, whereas their corresponding 

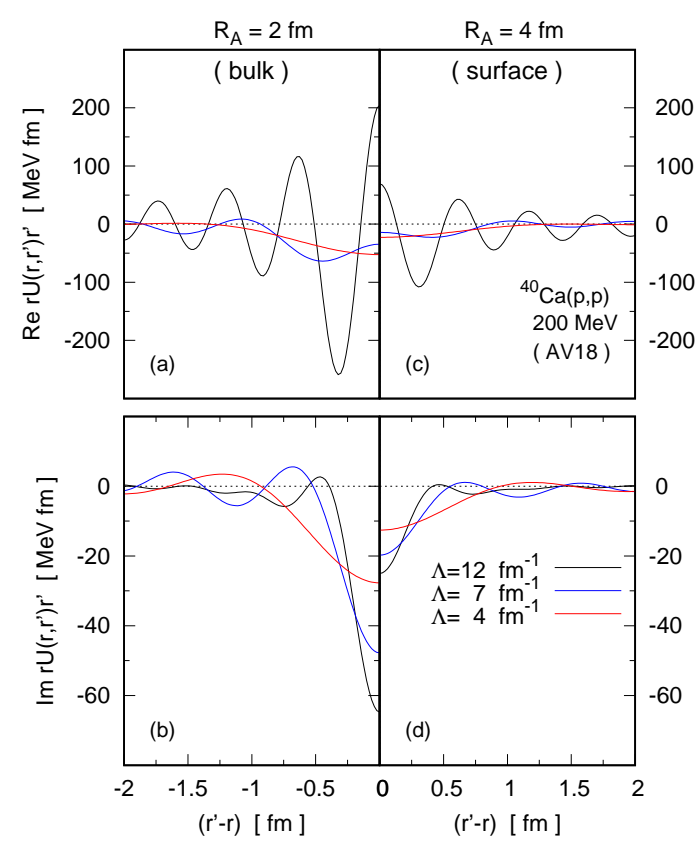

FIG. 5. Nonlocal behavior of the $s$-wave $r^{\prime} U\left(r^{\prime}, r\right) r$ as function of $\left(r^{\prime}-r\right)$, satisfying $r+r^{\prime}=$ constant. Black, blue and red curves correspond to results for $\Lambda=12,7$ and $4 \mathrm{fm}^{-1}$, respectively. See text for descriptions of each panel.

absorptive parts are very similar. Note also that all components feature damped oscillations as $\left|r-r^{\prime}\right|$ increases. The question that arises at this stage is whether differences in the nonlocal structure of these potentials, as driven by the cutoff $\Lambda$, have any incidence on the calculated scattering observables.

\section{B. Invariability of scattering observables}

A test of the equivalence of $U_{\Lambda}$ in coordinate space, for $\Lambda$ above its critical value, comes from the actual calculation of scattering observables. In this case we solve the integral equation for the scattering wavefunction

$$
\begin{aligned}
u_{j l}(r)= & \frac{1}{k} F_{l}(k r)+\left(\frac{2 m}{\hbar^{2}}\right) \iint d r^{\prime} d r^{\prime \prime} \\
& G_{l}^{(+)}\left(r, r^{\prime} ; k\right) r^{\prime} U_{j l}^{[s]}\left(r^{\prime}, r^{\prime \prime}\right) r^{\prime \prime} u_{j l}\left(r^{\prime \prime}\right),
\end{aligned}
$$

leading to exact solutions for the scattering waves in the presence of Coulomb interaction [26. Here $F_{l}$ denotes the regular Coulomb function for partial wave $l, j$ the total angular momentum, and $k$ the momentum of the projectile in the $N A$ center of momentum reference frame. The propagator $G_{l}^{(+)}\left(r, r^{\prime} ; k\right)$ represents the Green's function associated to the Coulomb interaction of point particles
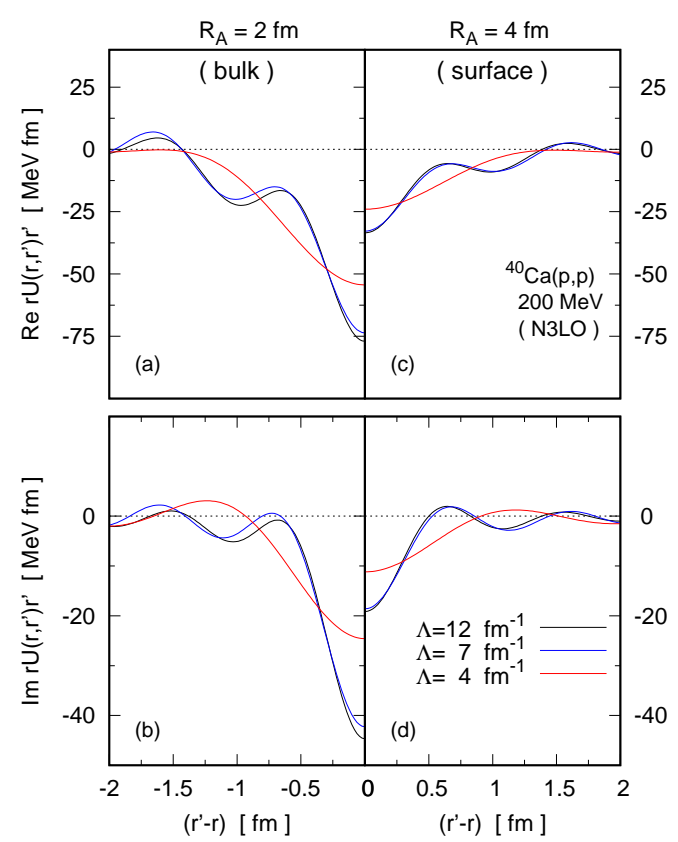

FIG. 6. The same as Fig. 5 but for N3LO.

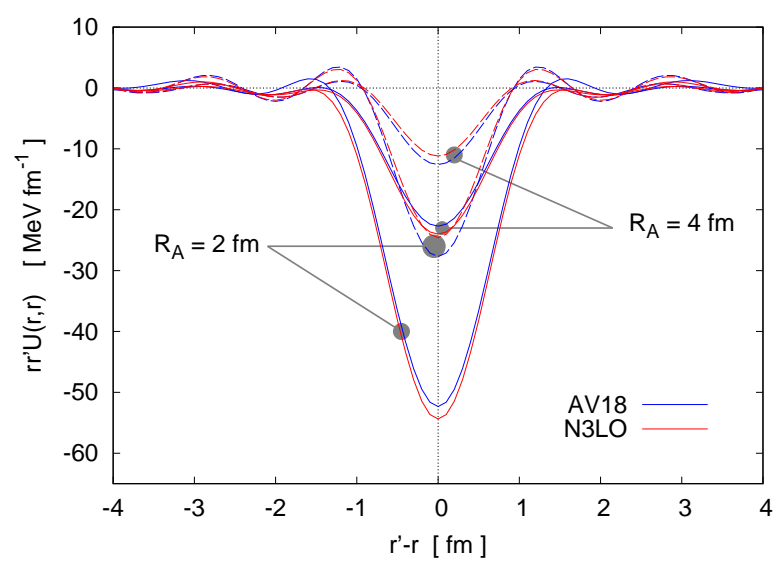

FIG. 7. Nonlocal behavior of the $s$-wave optical potential at bulk and surface vicinities in the case of $\Lambda=4 \mathrm{fm}^{-1}$. The optical potential corresponds to $p+{ }^{40} \mathrm{Ca}$ elastic scattering at $200 \mathrm{MeV}$ beam energy. Blue and red curves denote results based on AV18 and N3LO bare interactions, respectively.

of charge $Z e$ (nucleus) and $e$ (incident proton) [27]. Furthermore, $U^{[s]}$ consists of the (nonlocal) optical potential superposed to the Coulomb-screened interaction, namely

$$
r^{\prime} U_{j l}^{[s]}\left(r^{\prime}, r\right) r=r^{\prime} U_{j l}\left(r^{\prime}, r\right) r+\left[V_{C}(r)-\frac{Z e^{2}}{r}\right] \delta\left(r^{\prime}-r\right),
$$


resulting a nonlocal finite range interaction. Here $V_{C}(r)$ represents the electrostatic interaction between the charge distribution of the nucleus and the charge of the projectile. The hadronic component itself is composed by its central and spin-orbit contributions as

$$
U_{j l}=U_{l}^{(c)}+\langle\boldsymbol{\sigma} \cdot \boldsymbol{\ell}\rangle_{j l} U_{l}^{(s o)},
$$

with $\langle\boldsymbol{\sigma} \cdot \boldsymbol{\ell}\rangle_{j l}=[j(j+1)-l(l+1)-3 / 4]$.

In Fig. 8 we show the calculated differential cross section $(d \sigma / d \Omega)$ and analyzing power $\left(A_{y}\right)$ for proton elastic scattering from ${ }^{40} \mathrm{Ca}$ at $200 \mathrm{MeV}$ beam energy. Blue and red curves denote results based on AV18 and N3LO, respectively. Observables are calculated in coordinate space using four values for $\Lambda$. Three of these correspond to $\Lambda=12,7$ and $4 \mathrm{fm}^{-1}$, whose corresponding observables are all plotted with solid curves, becoming indistinguishable to the eye. The case $\Lambda=3.5 \mathrm{fm}^{-1}$ (dashed curves) has been chosen deliberately below the critical one in order to contrast its results with the other three values. As observed, all results with $\Lambda \geq 4 \mathrm{fm}^{-1}$ yield practically identical $d \sigma / d \Omega$ and $A_{y}$. Such is not the case for $\Lambda=3.5 \mathrm{fm}^{-1}$, resulting in weaker differential cross section and more structured $A_{y}$. Regarding the reaction cross section, all three cases with $\Lambda \geq 4 \mathrm{fm}^{-1}$ for AV18 (N3LO) yield $\sigma_{R}=540.0(524.9) \mathrm{mb}$, whereas for $\Lambda=3.5 \mathrm{fm}^{-1}$, we obtain $\sigma_{R}=505.0(490.8) \mathrm{mb}$. Differences of about $35 \mathrm{mb}$ are consistent with the trend observed for $d \sigma / d \Omega$ in Fig. 8 .

\section{Volume integrals}

As a means to check consistency of the results, we have calculated volume integrals of the central part of the optical potential in the two representations under study. In coordinate space we evaluate explicitly

$$
J=\int d \boldsymbol{r} d \boldsymbol{r}^{\prime} U_{c}\left(\boldsymbol{r}^{\prime}, \boldsymbol{r} ; E\right),
$$

with $U_{c}$ the central component of the optical potential. Considering that

$$
\tilde{U}_{c}\left(\boldsymbol{k}^{\prime}, \boldsymbol{k} ; E\right)=\frac{1}{(2 \pi)^{3}} \int d \boldsymbol{r} d \boldsymbol{r}^{\prime} e^{-i \boldsymbol{k}^{\prime} \cdot \boldsymbol{r}^{\prime}} U_{c}\left(\boldsymbol{r}^{\prime}, \boldsymbol{r} ; E\right) e^{i \boldsymbol{k} \cdot \boldsymbol{r}},
$$

it becomes evident that

$$
J=\left.8 \pi^{3} \tilde{U}_{c}\left(\boldsymbol{k}^{\prime}, \boldsymbol{k} ; E\right)\right|_{\boldsymbol{k}^{\prime}=\boldsymbol{k}=0}
$$

Thus, in momentum space the volume integral $J$ can be obtained from the potential matrix element at $k^{\prime}=$ $k=0$, unaffected by any finite cutoff with narrow width. In the case of the optical potential based on AV18 we obtain $J / A=-(536.2+i 225.9) \mathrm{MeV} \mathrm{fm}^{3}$ in momentum space, while in coordinate space we obtain for the four cutoffs $\Lambda, J / A=-(536.7+i 226.1) \mathrm{MeV} \mathrm{fm}^{3}$. In the case of results based on $\mathrm{N} 3 \mathrm{LO}$ we obtain $J / A=$

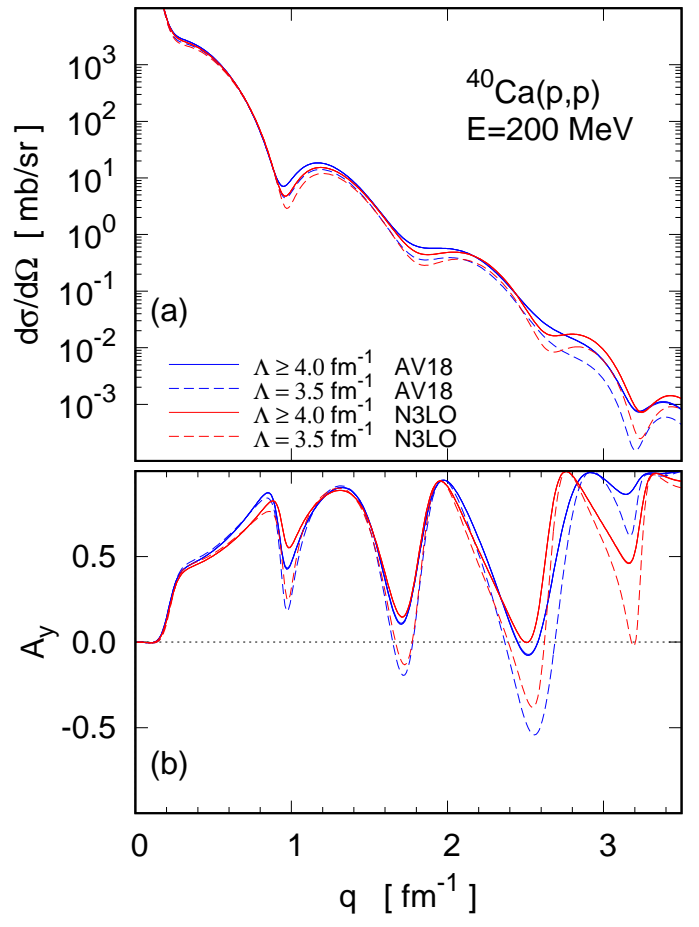

FIG. 8. Differential cross section (a) and analyzing power (b) as functions of the momentum transfer for ${ }^{40} \mathrm{Ca}(p, p)$ scattering at $200 \mathrm{MeV}$. Blue (red) curves correspond to results based on AV18 (N3LO) bare interaction. Solid curves correspond to $\Lambda=12,7$ and $4 \mathrm{fm}^{-1}$, whereas dashed curves correspond to $\Lambda=3.5 \mathrm{fm}^{-1}$.

$-(571.6+i 199.8) \mathrm{MeV} \mathrm{fm}^{3}$ in momentum space, while in coordinate space all four cutoffs yield $J / A=-(572.2+$ $i 200.0) \mathrm{MeV} \mathrm{fm}^{3}$. The differences among the two representations, close to $0.1 \%$, can be attained to the various numerical procedures involved in multipole evaluations and quadratures.

\section{NON-REDUCIBLE $k$-DOMAIN}

We have calculated momentum-space optical model potentials for proton elastic scattering off ${ }^{40} \mathrm{Ca}$ at several beam energies $E$ between 30 and $800 \mathrm{MeV}$. The procedures we follow are the same as the ones applied in the previous section. Considering that realistic $N N$ interactions are designed for nucleon beam energies of up to $\sim 300 \mathrm{MeV}$, applications for $E \geq 400 \mathrm{MeV}$ are based on procedures described in Ref. [18, where minimal relativity corrections are included in the evaluation of the optical potential. The same reference describes the inclusion of a separable term added to the bare interaction, in order to reproduce exactly empirical $N N$ scattering am- 
plitudes above pion production threshold. Apart from these considerations, the folding structure of the optical potential at these energies are the same as described in Sec. III. For consistency, we have excluded the N3LO bare potential from applications at these high energies.

Once the $N A$ potentials are evaluated we proceed to calculate their scattering observables with various choices of cutoff $\Lambda$, aiming to identify domains that yield the same results within a given accuracy. In Fig. 9 where we plot reaction cross sections $\sigma_{R}$ for $p+{ }^{40} \mathrm{Ca}$ elastic scattering as functions of $\Lambda$. Numerical labels indicate the beam energy in $\mathrm{MeV}$ units, while blue and red curves denote results based on AV18 and N3LO bare interactions, respectively. We observe that all curves for $\sigma_{R}$ as functions of $\Lambda$ reach a plateau above certain critical value, which we denote $\Lambda_{A}$. The subscript $A$ is intended to distinguish this cutoff, applied to $N A$ optical potentials, from those used in renormalization group transformations applied to $N N$ potentials. Thus, for a given energy, if we apply a cutoff to the momentum-space potential at or above its corresponding $\Lambda_{A}$, the scattering observables remain unaffected. This property leads to the notion that there is a minimum cutoff which renders $N A$ scattering observables unchanged. We shall refer to the complement of that interval as non-reducible $k$-domain. Cutoffs within the non-reducible $k$-domain alter the scattering observables associated to the optical potential under consideration. As we have seen in the previous section, different choices of $\Lambda$ above $\Lambda_{A}$ yield different coordinate-space structure, with the case $\Lambda=\Lambda_{A}$, resulting in the least structured potential. This feature hints us a means to compare on an equivalent footing nonlocal structures of different potentials. Henceforth, potential with cutoff at $\Lambda_{A}$ shall referred as reduced potentials, with its implied nonlocal behavior identified as reduced nonlocality.

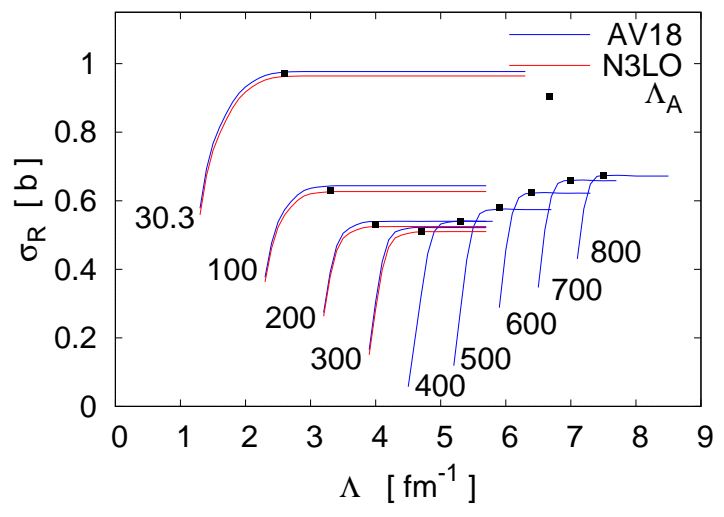

FIG. 9. Reaction cross section as a function of the cutoff $\Lambda$ applied to the optical model potential for $p+{ }^{40} \mathrm{Ca}$ elastic scattering. Black and red curves represent results based on AV18 and N3LO bare interactions. Labels on each curve refer to the beam energy. Blue squares denote the critical cutoff.

Examining more closely Fig. 9 for $\sigma_{R}$ we observe that

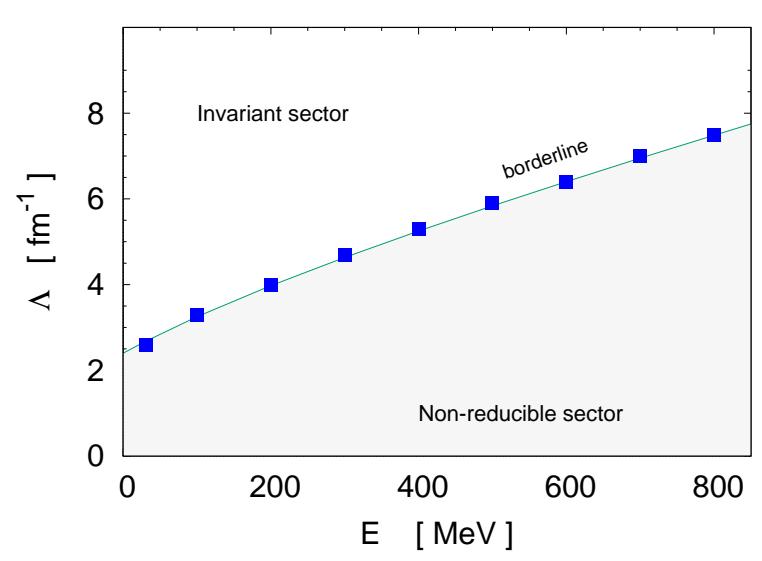

FIG. 10. Critical cutoff $\Lambda_{A}$ for the non-reducible $k$-domain as function of the beam energy $E$ in the case of proton elastic scattering from ${ }^{40} \mathrm{Ca}$.

the position of $\Lambda_{A}$ increases monotonically with the energy. To obtain $\Lambda_{A}$ at the edge of the plateau we look for the smallest $\Lambda$ which satisfies

$$
\left|\frac{\partial \sigma_{R}}{\partial \Lambda}\right| \leq \varepsilon
$$

with $\varepsilon=0.01 \mathrm{mb} \mathrm{fm}$. In Fig. 10 we plot with filled squares the obtained values of $\Lambda_{A}$. We have found that this behavior can be characterized by means of the formula

$$
\Lambda_{A}(E)=\sqrt{\Lambda_{0}^{2}+k_{E}^{2}},
$$

which is plotted with solid curve (borderline). In this case $\Lambda_{0}=2.4 \mathrm{fm}^{-1}$, with $k_{E}$ the relative momentum in the center of momentum reference frame associated to the beam energy $E$. The region below the borderline corresponds to the non-reducible $k$-domain, while the region above it represents the sector which yield scattering observables invariant. Eq. 12 for $\Lambda_{A}$ has to be taken as an estimate of the borderline since it depends on the criteria set for $\varepsilon$, width $\delta$ of the cutoff as well as numerical accuracy. This borderline may also depend on the optical model as well as nature of the $N N$ effective interaction under use. Its extension to proton collisions from targets other than ${ }^{40} \mathrm{Ca}$ is beyond the scope of this study.

\section{A. $p+{ }^{40}$ Ca scattering at $30.3 \mathrm{MeV}$}

We now study proton scattering from ${ }^{40} \mathrm{Ca}$ at 30.3 $\mathrm{MeV}$, an energy which allows us to extend the previous discussion to other documented nonlocal optical potentials developed for $p+{ }^{40} \mathrm{Ca}$ elastic scattering [15, 16]. Apart from the AV18- and N3LO-based optical potentials, we include results based on the microscopic optical 
model of Ref. [16] within the NSM based on Green's function formalism in the Random-Phase Approximation using Gogny's effective interaction. Here we also consider the Perey-Buck-type parametrization reported by Tian et al. [15], phenomenological approach of common use.

The last two potentials are calculated in coordinate space, with its equivalent central and spin-orbit counterparts in momentum space obtained from

$$
\tilde{U}_{l}\left(k^{\prime}, k\right)=\frac{2}{\pi} \int_{0}^{\infty} r^{\prime 2} d r^{\prime} \int_{0}^{\infty} r^{2} d r j_{l}\left(k^{\prime} r^{\prime}\right) U_{l}\left(r^{\prime}, r\right) j_{l}(k r) .
$$

In order to compare $N A$ potentials on a same footing we look for their reduced form. Hence, starting with a potential in coordinate space we transform it to momentum space using Eq. (13), to then apply a momentum cutoff and transform it back to coordinate space. The transformed potential $U_{\Lambda}$ is then used to obtain its scattering observables solving Eq. (5).

The presence of local contributions $V_{l}(r)$ in the hadronic part of the $N A$ potential deserves special attention in the procedure outlined above. This feature takes place in the NSM approach, where the HartreeFock term is local. This also occurs for the direct term in coordinate-space folding models based on local effective interactions 6]. In such cases their momentum-space representation becomes

$$
\tilde{V}_{l}\left(k^{\prime}, k\right)=\frac{2}{\pi} \int_{0}^{\infty} r^{2} d r j_{l}\left(k^{\prime} r\right) V_{l}(r) j_{l}(k r) .
$$

What is interesting in this case is that its transformation back to coordinate space, after the application of a cutoff at $\Lambda$, result into a nonlocal potential $V_{\Lambda}\left(r^{\prime}, r\right)$. Hence, to the extent there is no sensitivity to the cutoff, local potentials also have nonlocal equivalents. This trend is somewhat observed in panels $a^{\prime}$ of Figs. 3 and 4 , where the sharp diagonal structure for the imaginary part of the potential (resembling locality) loses its sharpness as the cutoff is diminished. For $\Lambda=4 \mathrm{fm}^{-1}$ the equivalent potential is clearly nonlocal.

In Fig. 11 we plot calculated differential cross section $d \sigma / d \Omega$ (a) and analyzing power $A_{y}$ (b) as functions of the center-of-mass scattering angle $\theta_{c . m}$., for proton scattering off ${ }^{40} \mathrm{Ca}$ at $30.3 \mathrm{MeV}$ beam energy. The data are taken from Refs. [28, 29, included here in order to visualize the level of agreement of each approach. Blue and red curves denote results based on AV18 and N3LO bare interactions, respectively. Black curves represent results based on the NSM microscopic approach using Gogny's effective interaction. Green curves correspond to results based on the Perey-Buck-type nonlocal parametrization of Ref. [15. In this figure we plot results with cutoffs at $\Lambda=7,4$ and $2.6 \mathrm{fm}^{-1}$, resulting in nearly full overlap among curves of each case. These results illustrate the level at which different cutoffs above $\Lambda_{A}$ result in the same observables. The differences among the four approaches indicate the extent to which their corresponding reduced potentials differ from each other.

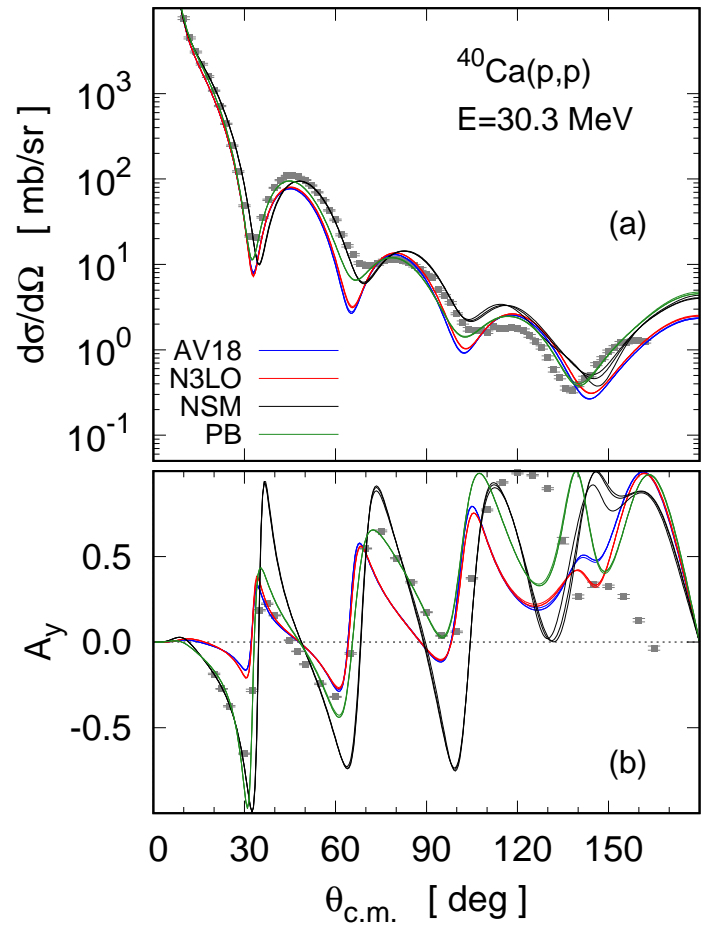

FIG. 11. Differential cross section (a) and analyzing power (b) as functions of the center-of-mass scattering angle for ${ }^{40} \mathrm{Ca}(p, p)$ scattering at $30.3 \mathrm{MeV}$. Overlapping solid curves correspond to results based on $\Lambda=6,4$ and $2.6 \mathrm{fm}^{-1}$. Data from Ref. 28, 29].

In Figs. 1215 we show contour plots in the $\left(r, r^{\prime}\right)$ plane for the calculated $s$-wave $r^{\prime} U\left(r^{\prime}, r\right) r$. Panels $a, b$ and $c$ correspond to $\Lambda=6,4$ and $2.6 \mathrm{fm}^{-1}$, respectively. All cases meet the criteria $\Lambda \geq \Lambda_{A}$, resulting in different nonlocal structure but sharing the same scattering observables. On each Fig. panels on the l.h.s. (r.h.s.) correspond to their real (imaginary) parts. Plots in Figs. 12 and 13 are based on AV18 and N3LO bare potentials, respectively. Plots in Fig. 14 are based on NSM microscopic approach, while Fig. 15 is based on PereyBuck-type parametrization of Ref. [15]. Note that the scale of panels $a$ in Figs. 1215 range from $-100 \mathrm{MeV}$ up to zero. In order to make comparable these Figs. all corresponding panels use the same scale. Panels $c$ show the reduced potential since the cutoff applied in these cases corresponds to $\Lambda_{A}$ given by Eq. (12). We observe the following:

a.- All upper frames $a$ and $a^{\prime}$ show the nonlocal behavior of the potential closest to its original form. By closest we mean they all have been already affected by a cutoff, though relatively large with respect to the critical one. Observing panel $a$ for AV18 we identify a 

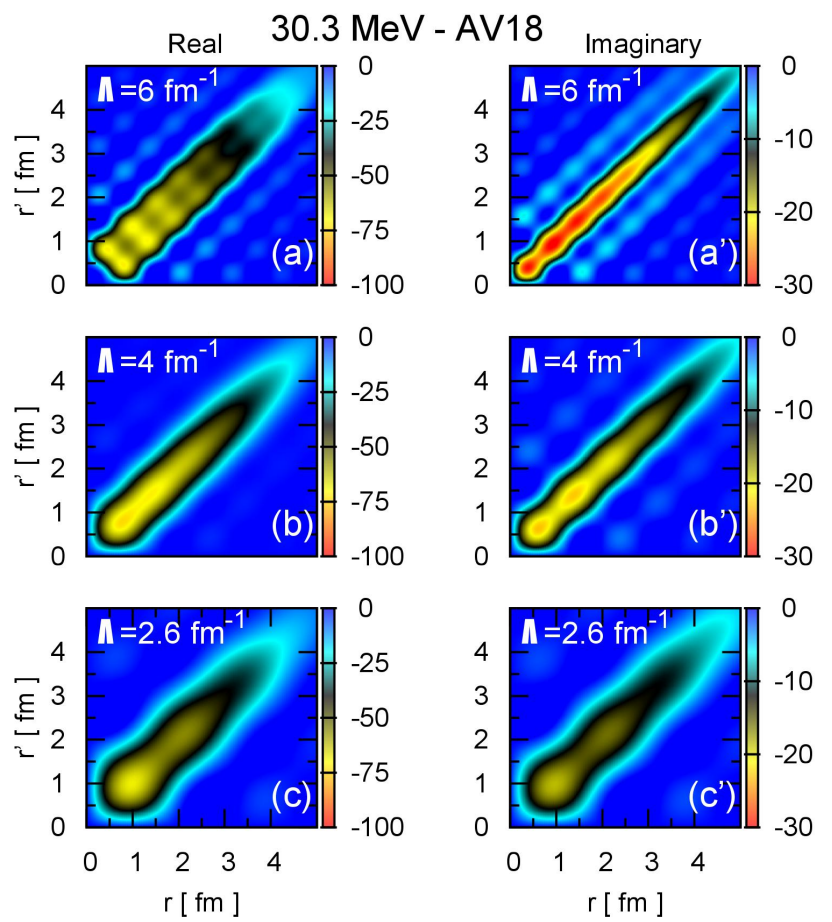

FIG. 12. Contour plots for the real (1.h.s. panels) and imaginary (r.h.s. panels) $s$-wave central optical potential as functions of the relative distance $r$ and $r^{\prime}$. Potential for $p+{ }^{40} \mathrm{Ca}$ scattering at $30.3 \mathrm{MeV}$. Results based on AV18 bare interaction. Frames a, b and c represent results based on $\Lambda=6$, 4 and $2.6 \mathrm{fm}^{-1}$, respectively. All three potentials yield the same scattering observables. Color bar in units of $\mathrm{MeV} \mathrm{fm}^{-1}$.
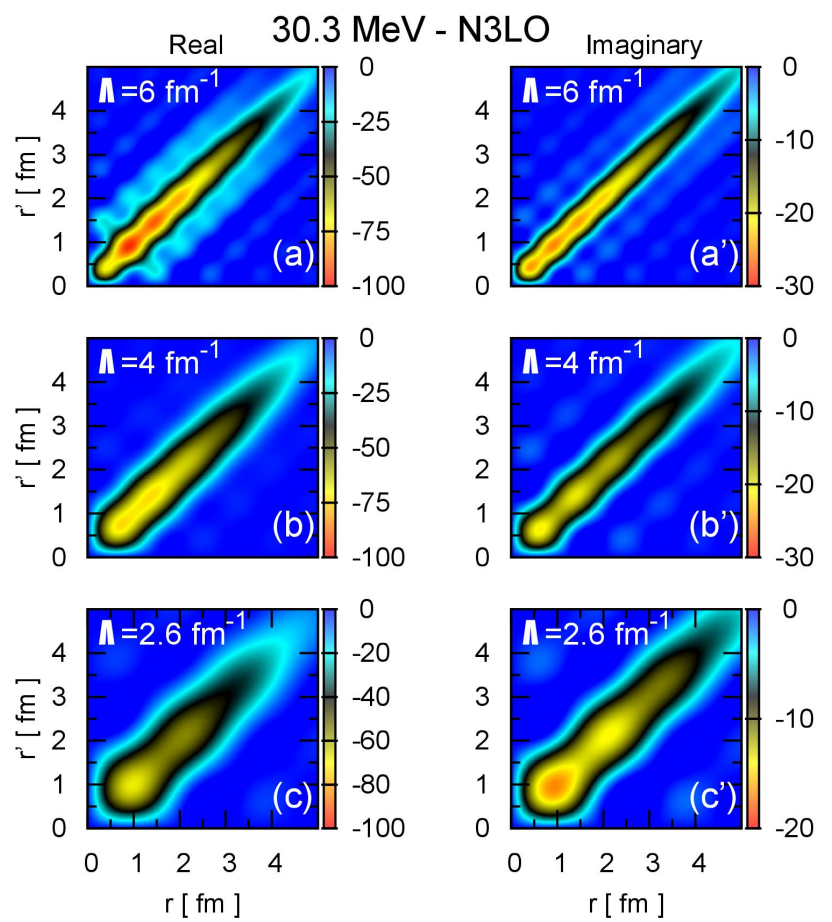

FIG. 13. The same as Fig. 12, but for N3LO bare interaction. rather flat diagonal band of about $\sim 1$ fm width, with strength nearing $-75 \mathrm{fm}$. This band, which does not appear in the other cases, weakens near the surface $\left(r \approx r^{\prime} \approx 4 \mathrm{fm}\right)$. Frames $a$ for N3LO and NSM exhibit sharper potentials along the diagonal, vanishing around $4 \mathrm{fm}$. The corresponding panel for PB shows a weaker and smooth potential with an elongated oval shape. With the exception of Fig. 15 for PB, all potentials exhibit fine structures in the form of regular spots away from the diagonal.

b.- As the cutoff $\Lambda$ diminishes, the coordinate-space potential becomes less structured. This is observed in all four cases. In this regard note how similar is the reduced potential based on AV18 with that based on the N3LO bare interaction. In the case of NSM in Fig. 14 the real part of the reduced potential is more shallow at the center than in the previous ones, also weakening as the surface is reached at $r \sim r^{\prime} \approx 2 \mathrm{fm}$. In the case of $\mathrm{PB}$, the real part resembles very much the ones based on NSM.

c.- The imaginary part of the potentials shown at the r.h.s. of each Fig. based on AV18 and N3LO exhibits minor differences, both in their raw and reduced forms. The other two cases, namely NSM and PB are much different. The former starts at frame $a$ ' with a narrow band and end up at panel $c$ ' with a less structures absorptive component confined to radii below $2 \mathrm{fm}$. The opposite occurs for $\mathrm{PB}$, where the absorptive part takes place above radii of $3 \mathrm{fm}$, remaining in frame $c$ ' away from the bulk of the nucleus.

\section{B. Reduced potentials in coordinate space}

As discussed in the previous section, the critical cutoff $\Lambda_{A}$ lead to optical potentials with least structure in coordinate space. This characteristic is observed in all partial waves as well as energies. In order to illustrate this feature in $3 \mathrm{D}$ surface plots, we consider $s$-wave potentials (central part) for $p+{ }^{40} \mathrm{Ca}$ elastic scattering at 800, 200 and $30.3 \mathrm{MeV}$. In Fig. 16 we show surface plots of the real (l.h.s. graphs) and imaginary (r.h.s. graphs) $s$-wave reduced central optical potential $r U\left(r, r^{\prime}\right) r^{\prime}$, as functions of $r$ and $r^{\prime}$. These results are based on AV18 bare interaction. The critical cutoffs for each case are 8, 4, and $2.6 \mathrm{fm}^{-1}$, respectively. Color bars and vertical axes are in units of $\mathrm{MeV} \mathrm{fm}^{-1}$. As observed, all potentials exhibit pronounced structures in both real and imaginary components. The case of $30.3 \mathrm{MeV}$ appears the most smooth of all. As the energy increases, the potential features strong oscillating patterns near and away of the diagonal. We have to stress that all these potentials are the least structured in coordinate space, consistent with the scattering observables they have in their original form, namely with large cutoff.

For completeness in this discussion, in Fig. 17 we plot 
the differential cross section (a) and analyzing power (b) as functions of the momentum transfer for ${ }^{40} \mathrm{Ca}(p, p)$ scattering at $800 \mathrm{MeV}$. Here overlapping solid curves correspond to results based on $\Lambda=12,10$ and $8 \mathrm{fm}^{-1}$. Dashed curved correspond to $\Lambda=7.5 \mathrm{fm}^{-1}$, below the critical value. The data are taken from Refs. 30, 31]. As observed, this case exhibits the same features as the ones discussed in the previous sections, namely there is range of cutoffs above $\Lambda_{A}$ which do not alter the calculated scattering observables. The case $\Lambda=7.5 \mathrm{fm}^{-1}$, below $\Lambda_{A}$, displays clearly its differences with the actual observables. The specific issue on the level of agreement of the model with the data requires a more dedicated study which is beyond the scope of this work. Regardless on any change at the level of the microscopic description at this energy, it is safe to state that the nonlocal structure of the calculated potential will remain essentially the same as the one shown in Fig. 16

\section{SUMMARY AND CONCLUSIONS}

We have investigated the nonlocal structure of optical model potentials for $N A$ based on microscopic approaches. To this purpose, in-medium folding optical potentials have been calculated in momentum space and their corresponding coordinate-space counterpart have been examined, focusing our attention on their nonlocal shape. The $N N$ effective interaction consists of the actual full off-shell $g$ matrix in Brueckner-Hartree-Fock approximation. The nonlocality of effective interactions is preserved throughout all the stages of the the calculation. The bare interactions under consideration are Argonne $v_{18}$ potential and chiral next-to-next-to-next-toleading order bare interaction. The study has been focused on proton elastic scattering off ${ }^{40} \mathrm{Ca}$ at beam energies between 30 and $800 \mathrm{MeV}$. Applications at $30.3 \mathrm{MeV}$ have also been studied using the microscopic NSM optical potential as well as phenomenological Perey-Buck type. We have found that the gradual suppression of high-momentum contributions of the optical potential results in quite different-looking coordinate-space counterparts. Despite this non-uniqueness in their nonlocal structure, the implied scattering observables remain unchanged for momentum cutoff above a critical one, which depends on incident energy of the projectile.

Folding optical model potentials based on any realistic $N N$ interaction, as the one used in this work, offer the possibility to explore in a systematic way nucleon collisions at energies ranging from tens of $\mathrm{MeV}$ up to near
$\mathrm{GeV}$ energies. A crucial point in the choice of this framework is the fact that all sources of nonlocalities are preserved throughout, namely the determination of the effective $N N$ interaction and also the evaluation of the potential itself. Thus, momentum-space in-medium folding potentials constitute a general microscopic starting point to investigate the optical model and its nonlocal features. In this framework the potential is evaluated in momentum space, representation which enables the treatment of nonlocalities in a natural way. In this regard the model is not a particular one, but instead a general one to account for these features.

The identification of an energy-dependent critical cutoff that guarantees invariability in the scattering observables is an important finding in this work, particularly due to its implications on the coordinate-space structure of equivalent potentials. We have demonstrated that the nonlocal shape of the potential becomes less structured as the cutoff is decreased until a critical value, which guarantees that scattering observables remain unchanged. An immediate implication of this result is that any attempt to quantify the degree of nonlocality of a given potential is meaningless. The non uniqueness of nonlocality in coordinate space also questions the systematic resort to Gaussian nonlocalities in the design of phenomenological potentials, as first introduced by Perey and Buck in the 60s. Despite this limitation, we have found that potentials constructed in any representation, even local ones in coordinate space, can be subject to momentum cutoff which render the same scattering observables. The resulting potential in coordinate space is always nonlocal, although less structured when the cutoff is at $\Lambda_{A}$. This feature may shed light in works aiming to generate phenomenological nonlocal optical potentials.

From a practical point of view, the identification of the energy-dependence of the critical cutoff $\Lambda_{A}$ allows to identify beforehand the range of momenta relevant in the evaluation of an optical potential in momentum space. From the present study we can safely state that momentum components of the optical potential above $\Lambda_{A}$ are irrelevant. The generalization of this empirical law for $\Lambda_{A}$ to any mass number $A$ in the nuclear chart constitutes an extension of this work.

\section{ACKNOWLEDGMENTS}

H.F.A. thanks colleagues at CEA, Bruyères-le-Châtel, France, for their hospitality during his stay where part of this work was done.
[1] F. Perey and B. Buck, Nucl. Phys. 32, 353 (1962).

[2] J. S. Bell and E. J. Squires, Phys. Rev. Lett. 3, 96 (1959)

[3] J. P. Jeukenne, A. Lejeune, and C. Mahaux, Phys. Rep. 25, 83 (1976).
[4] L. Ray, G. W. Hoffmann, and W. R. Coker, Phys. Rep. 212, 223 (1992).

[5] W. H. Dickhoff and D. Van Neck, Many-Body Theory Exposed! (World Scientific, Singapore, 2008). 
[6] K. Amos, P. J. Dortmans, H. V. von Geramb, S. Karataglidis, and J. Raynal, Advances in Nuclear Physics, Advances in Nuclear Physics, Vol. 25 (Springer, New York, 2000).

[7] F. J. Aguayo and H. F. Arellano, Phys. Rev. C 78, 014608 (2008)

[8] M. Vorabbi, P. Finelli, and C. Giusti, Phys. Rev. C 93, 034619 (2016)

[9] H. V. von Geramb, The Interaction Between Medium Energy Nucleons in Nuclei (American Institute of Physics, New York, 1983).

[10] C. Elster, T. Cheon, E. F. Redish, and P. C. Tandy, Phys. Rev. C 41, 814 (1990)

[11] R. Crespo, R. C. Johnson, and J. A. Tostevin, Phys. Rev. C 41, 2257 (1990).

[12] H. F. Arellano, F. A. Brieva, and W. G. Love, Phys. Rev. C 41, 2188 (1990).

[13] H. F. Arellano, F. A. Brieva, and W. G. Love, Phys. Rev. C 52, 301 (1995)

[14] W. H. Dickhoff, R. J. Charity, and M. H. Mahzoon, Journal of Physics G: Nuclear and Particle Physics 44, 033001 (2017)

[15] Y. Tian, D.-Y. Pang, and Z.-Y. Ma, International Journal of Modern Physics E 24, 1550006 (2015).

[16] G. Blanchon, M. Dupuis, H. F. Arellano, and N. Vinh Mau, Phys. Rev. C 91, 014612 (2015).

[17] J. Rotureau, P. Danielewicz, G. Hagen, F. M. Nunes, and T. Papenbrock, Phys. Rev. C 95, 024315 (2017).

[18] H. F. Arellano and H. V. von Geramb, Phys. Rev. C 66, 024602 (2002)

[19] R. B. Wiringa, V. G. J. Stoks, and R. Schiavilla, Phys.
Rev. C 51, 38 (1995).

[20] D. R. Entem and R. Machleidt, Phys. Rev. C 68, 041001 (2003).

[21] H. F. Arellano and J.-P. Delaroche, Eur. Phys. Jour. A 51, 7 (2015).

[22] F. Isaule, H. F. Arellano, and A. Rios, Phys. Rev. C 94, 034004 (2016)

[23] Arellano, H. F., Isaule, F., and Rios, A., Eur. Phys. J. A 52, 299 (2016).

[24] J. Dechargé and D. Gogny, Phys. Rev. C 21, 1568 (1980).

[25] S. Bogner, R. Furnstahl, and A. Schwenk, Progress in Particle and Nuclear Physics 65, 94 (2010).

[26] H. F. Arellano and G. Blanchon, "Exact scattering waves off nonlocal potentials with coulomb interaction," (2018), arXiv:1806.05006

[27] H. F. Arellano and W. G. Love, Phys. Rev. C 76, 014616 (2007)

[28] B. Ridley and J. Turner, Nuclear Physics 58, 497 (1964)

[29] V. Hnizdo, O. Karban, J. Lowe, G. W. Greenlees, and W. Makofske, Phys. Rev. C 3, 1560 (1971)

[30] L. Ray, G. W. Hoffmann, M. Barlett, J. McGill, J. Amann, G. Adams, G. Pauletta, M. Gazzaly, and G. S. Blanpied, Phys. Rev. C 23, 828 (1981)

[31] G. Igo, G. Adams, T. Bauer, G. Pauletta, C. Whitten, A. Wreikat, G. Hoffmann, G. Blanpied, W. Coker, C. Harvey, R. Liljestrand, L. Ray, J. Spencer, H. Thiessen, C. Glashausser, N. Hintz, M. Oothoudt, H. Nann, K. Seth, B. Wood, D. McDaniels, and M. Gazzaly, Physics Letters B 81, 151 (1979). 

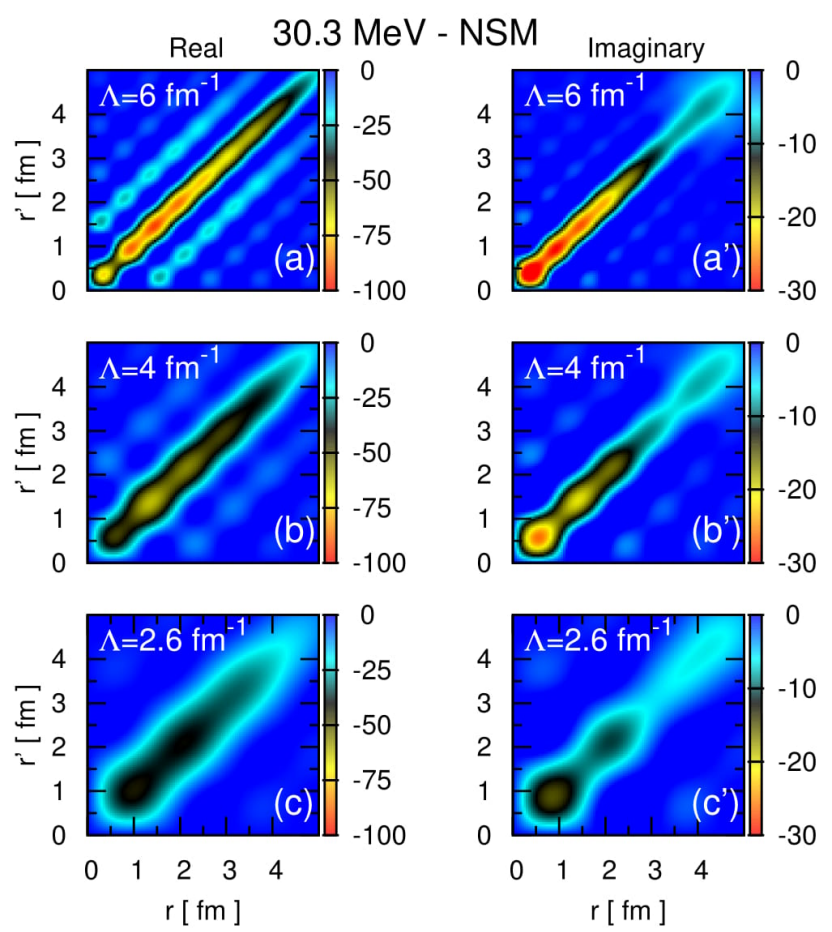

FIG. 14. The same as Fig. 12 but based on NSM approach.
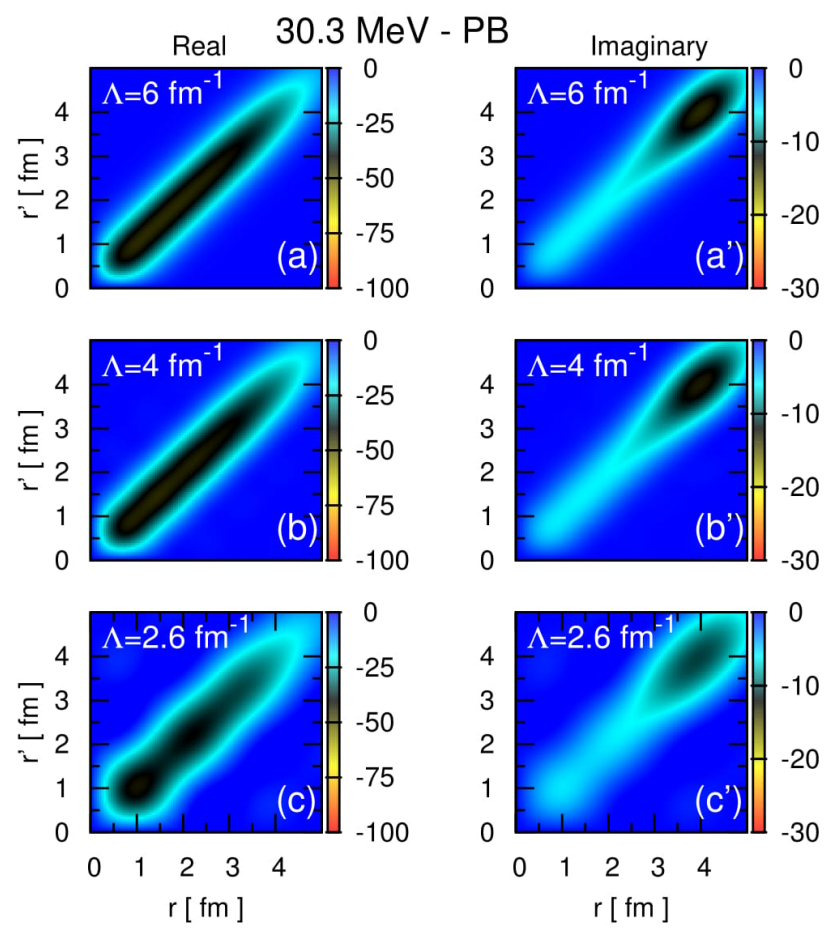

FIG. 15. The same as Fig. 12, but based on Perey-Bucktype nonlocal parametrization of Ref. [15. 


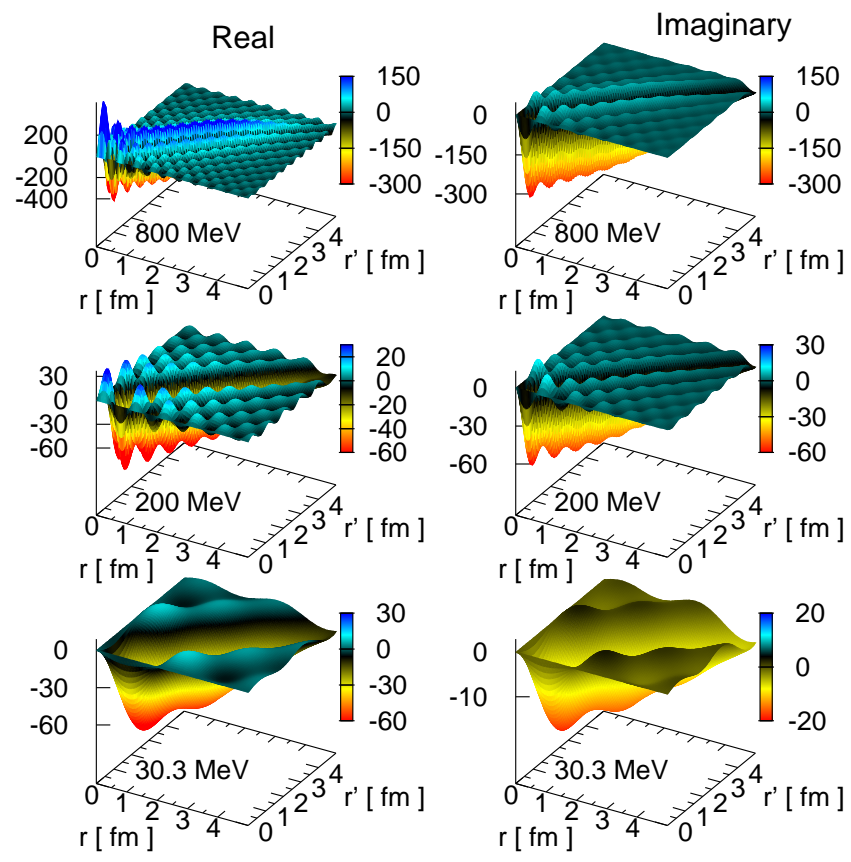

FIG. 16. Surface plots of real (1.h.s. graphs) and imaginary (r.h.s. graphs) $s$-wave reduced central optical potential $r U\left(r, r^{\prime}\right) r^{\prime}$ as functions of the relative distance $r$ and $r^{\prime}$. Potential for $p+{ }^{40} \mathrm{Ca}$ scattering at 800,200 and $30.3 \mathrm{MeV}$. Results based on AV18 bare interaction. Color bars and vertical axes are in units of $\mathrm{MeV} \mathrm{fm}^{-1}$. 


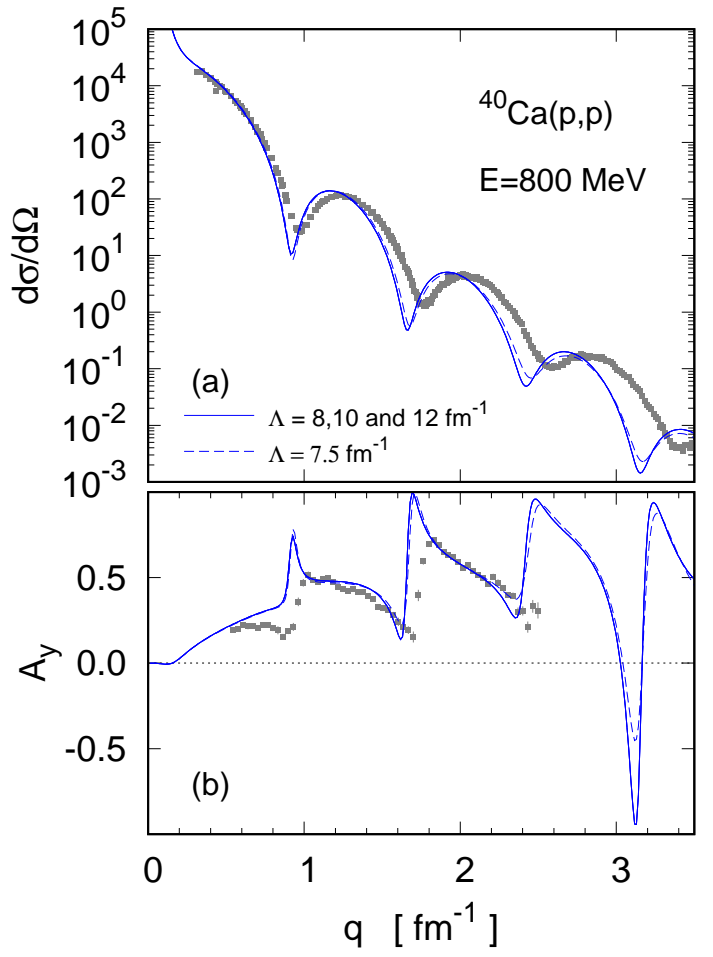

FIG. 17. Differential cross section (a) and analyzing power (b) as functions of the momentum transfer for ${ }^{40} \mathrm{Ca}(p, p)$ scattering at $800 \mathrm{MeV}$. Overlapping solid curves correspond to results based on $\Lambda=12,10$ and $8 \mathrm{fm}^{-1}$. Dashed curves correspond to $\Lambda=7.5 \mathrm{fm}^{-1}$. Data taken from Refs. 30, 31] 